\title{
Electromagnetic Induction in Three Dimensional Structures for Various Source Fields
}

\author{
R.D. Hibis and F.W. Jones \\ Department of Physics, and the Institute of Earth and Planetary Physics, \\ The University of Alberta, Edmonton, Alberta, Canada
}

(Received August 5, 1976; Revised October 26, 1977)

\begin{abstract}
A numerical method is used to calculate the electromagnetic fields associated with three-dimensional conductivity anomalies. Three source fieldconductivity structure combinations are considered. A uniform source above an island structure as well as a two-dimensional non-uniform source above an L-shaped embedded structure and a horizontal dipole above an embedded square anomaly are studied. Profiles of selected electromagnetic field quantities and ratios are presented along with the appropriate phases for various positions relative to the anomalous structure. Three dimensional figures of the amplitude of the electric and magnetic field components are given for each source field-conductivity structure combination. The source field effect is clearly evident in all profiles.
\end{abstract}

\section{Introduction}

The three-dimensional geomagnetic induction problem was first considered by Treumann (1970) who showed that the Green's tensor could be used to obtain a solution. Jones and PASCOE (1972), in an extension of their two-dimensional finite difference method obtained a solution for a three-dimensional buried conductivity inhomogeneity using a cubic mesh. This work was extended to grids of variable dimension by Lines and Jones (1973). Also, Weidelt (1975) used the Green's tensor method to calculate the electromagnetic induction effects of a three dimensional structure. The induction effects studied thus far for three-dimensional structures have been for uniform source fields. However, if the proper boundary conditions are applied to a finite difference mesh non-uniform source fields can be studied.

In this work three source and conductivity structure combinations are considered. The sources presented are one, two and three dimensional in the nature of the magnetic field calculated at the surface of a uniformly layered conductor. The conductivity structures, which differ for each source configuration used, are chosen to represent varying degrees of symmetry and conductivity contrasts. 
For each combination the source frequency is chosen such that the amplitudes of the electromagnetic field components presented will have large values.

\section{General Method of Solution}

The electromagnetic fields due to one, two and three-dimensional sources over layered laterally uniform conductors can be calculated by various methods. For the one-dimensional source the analytical solution for the layered earth as in Jones and PRICE (1970), is used here. For the two-dimensional source configuration the Fourier series method of HibBs and Jones (1976a, b) is used, and for the three-dimensional dipole source the method of RAMASWAMY (1973) is used. The electric field values thus calculated are used to provide boundary and initial conditions for the finite difference mesh of LINES and Jones (1973) and from this the electric fields due to three-dimensional embedded conductivity anomalies are determined. The magnetic field quantities are then obtained from the electric field solutions by using the appropriate finite difference equation.

\section{One-Dimensional Source Field}

The one-dimensional source field consists of a uniform sheet current flowing above a layered conductor. The boundary conditions for this case have been considered in detail by Jones and Price (1970). In the present work only the $E$-polarization case is considered. The electric field component is polarized in the $X$-direction and the magnetic field component is polarized in the $Y$-direction.

In the model considered, a uniform sheet current flows above a conductivity inhomogeneity embedded in a two-layered semi-infinite conducting region with a plane boundary. The first layer is $4 \mathrm{~km}$ in depth and of conductivity $4 \mathrm{mho} / \mathrm{m}$. The second layer extends to infinity and has conductivity $0.001 \mathrm{mho} / \mathrm{m}$. The embedded anomaly is a $6 \mathrm{~km}$ square intrusion of the lower conductive layer which penetrates the top layer to the surface of the conductor. The boundary conditions for the electric field are determined by the method of JONES and PRICE (1970) and the electric field solution over the anomaly is then found using the numerical technique of Lines and Jones (1973). This calculation was done for a frequency of $0.001 \mathrm{~Hz}$.

From the foregoing solution the profiles of $E_{x}, E_{y}, H_{z}, H_{z} / H_{y}$ and the apparent resistivity (calculated in the manner of CAGNIARD, 1953), as well as their respective phases were calculated for 3 profiles at different positions relative to the

anomaly as in Fig. 1a. The profiles were calculated over the range $-58 \mathrm{~km}$ to $+58 \mathrm{~km}$ in the $Y$ direction. Each field component or ratio with the exception 

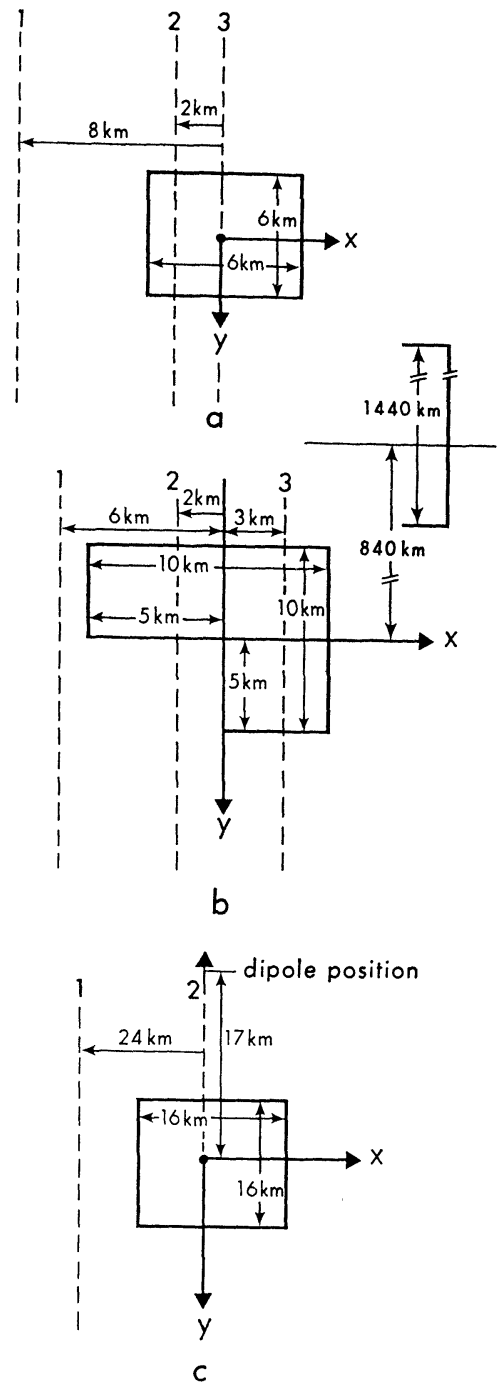

Fig. 1. Anomalous structures with profile positions $(1,2,3)$ indicated, a, anomaly associated with one-dimensional source; $b$, anomaly associated with two-dimensional source; c, anomaly associated with three-dimensional source. (not to scale)

of the apparent resistivity and $H_{z} / H_{y}$ was normalized with respect to its value at $-58 \mathrm{~km}$. All phases are normalized to zero at $-58 \mathrm{~km}$.

The first set of profiles is taken $8 \mathrm{~km}$ from the center of the anomaly in the negative $X$ direction and is given in Fig. 2. The normalized electric field amplitude $E_{x}$, exhibits a slight depression in the field due to the concentration of charge on the boundary of the anomaly. The normalized horizontal magnetic field $\left|H_{y}\right|$ is reasonably constant across this profile and changes by only about 4 percent. Normalized $\left|H_{z}\right|$ and therefore $H_{z} / H_{y}$ vary in the same manner over the anomaly due to the constant nature of $H_{y}$. Since both $\left|E_{x}\right|$ and $\left|H_{y}\right|$ are 


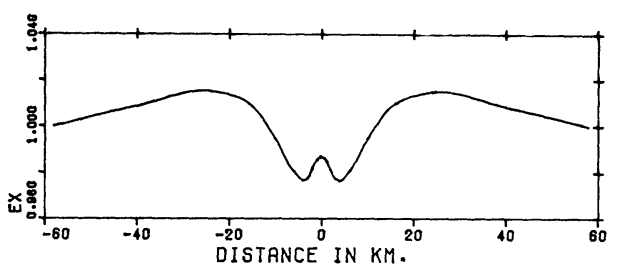

NORMALIZEO AMPLITUDE EX

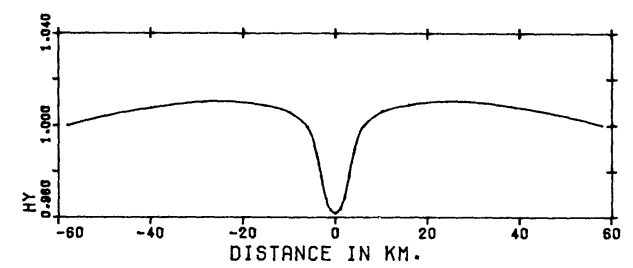

NORMALIZED AMPLITUDE HY

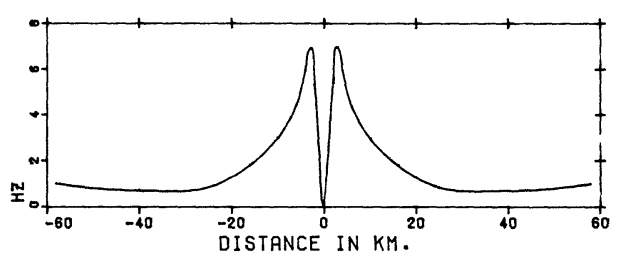

NORMALIZED AMPLITUDE $\mathrm{HZ}$

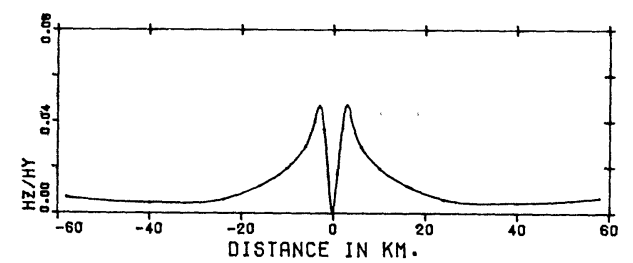

AMPLITUDE HZ/HY

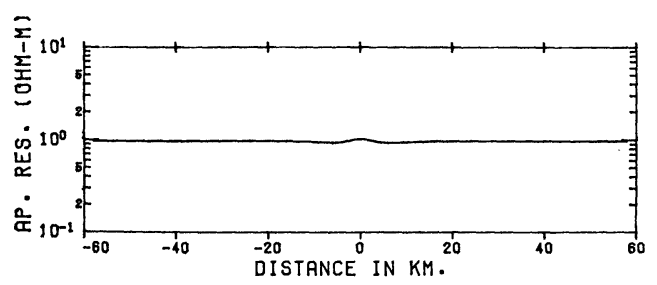

APPARENT RESISTIVITY

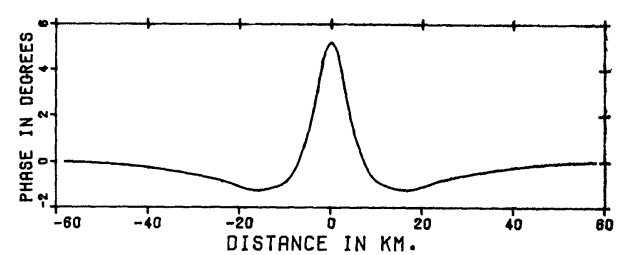

NORMALIZED PHASE OF EX

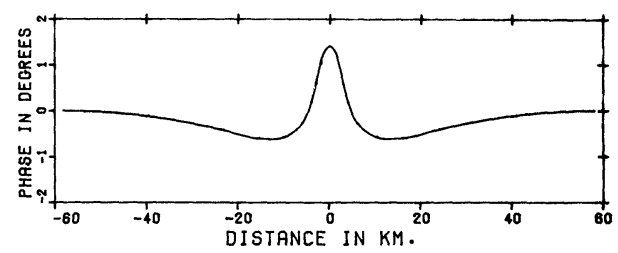

NORMALIZED PHASE OF HY

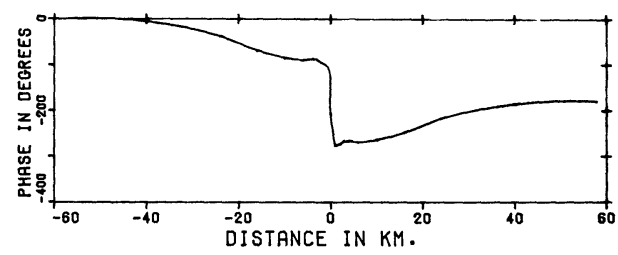

NORMALIZED PHASE OF $\mathrm{HZ}$

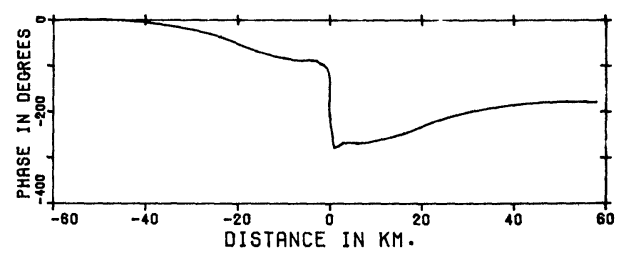

NORMALIZED PHASE OF $\mathrm{HZ/HY}$

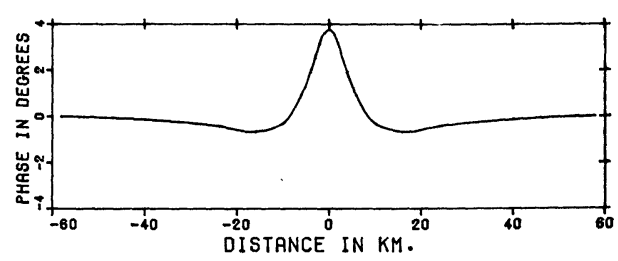

NORMALIZED PHASE OF EX/HY

Fig. 2. $\left|E_{x}\right|,\left|H_{y}\right|,\left|H_{z}\right|, H_{z} / H_{y}$ and $\rho_{a}$ profiles with normalized phases for one-dimensional source field and profile $1 .\left|E_{x}\right|,\left|H_{y}\right|$ and $\left|H_{z}\right|$ are normalized at $-58 \mathrm{~km}$. 
Electromagnetic Induction in Three Dimensional Structures for Various Source Fields

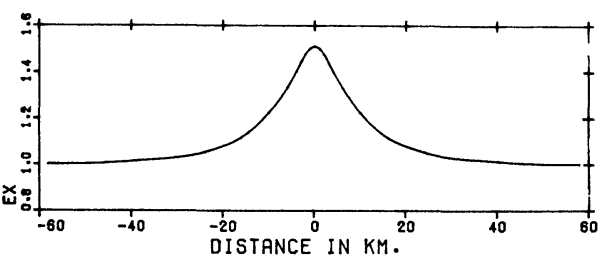

NORMALIZED AMPLITUDE EX

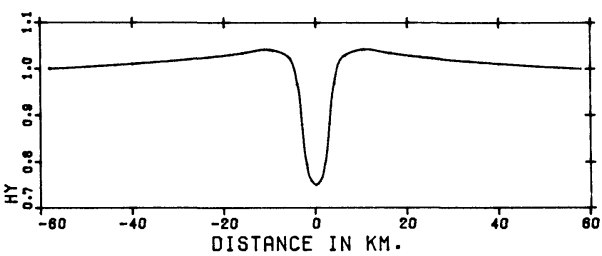

NORMALIZED AMPLITUDE HY

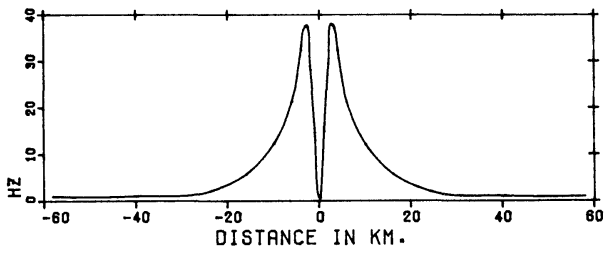

NORMALIZED AMPLITUDE HZ

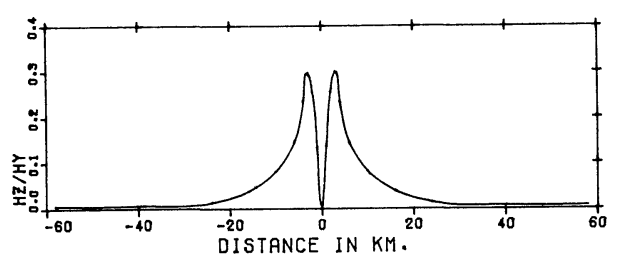

AMPLITUDE HZ/HY

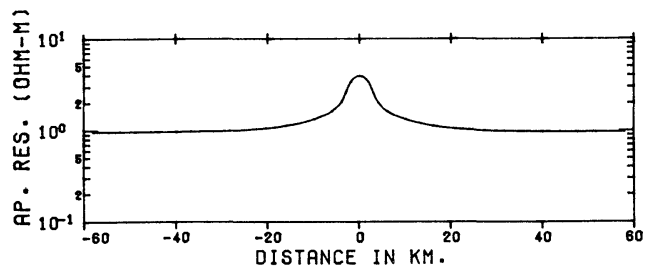

APPARENT RESISTIVITY

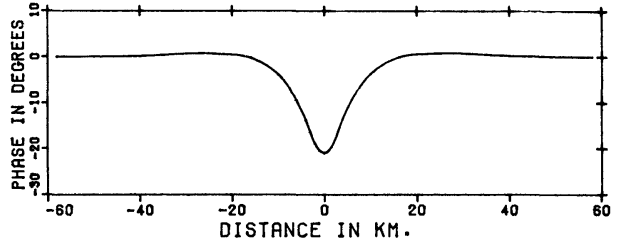

NORMALIZED PHASE OF EX

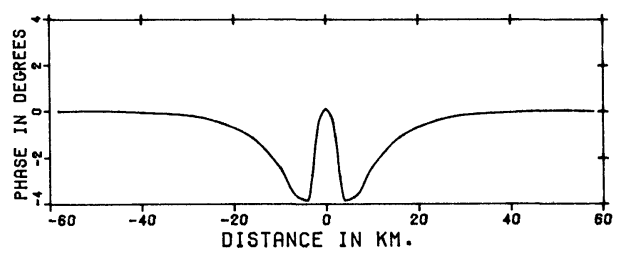

NORMALIZED PHASE OF HY

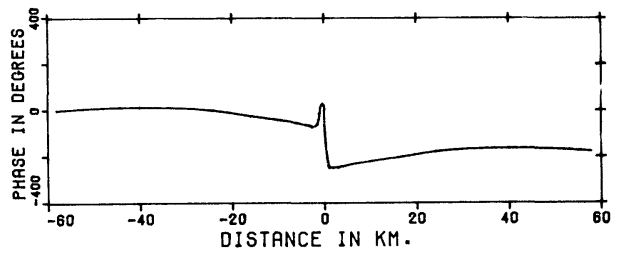

NORMALIZED PHASE OF $\mathrm{HZ}$

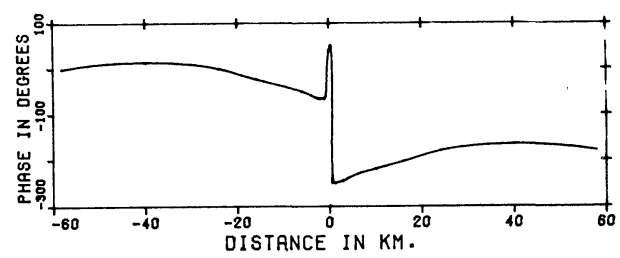

NORMALIZED PHASE OF HZ/HY

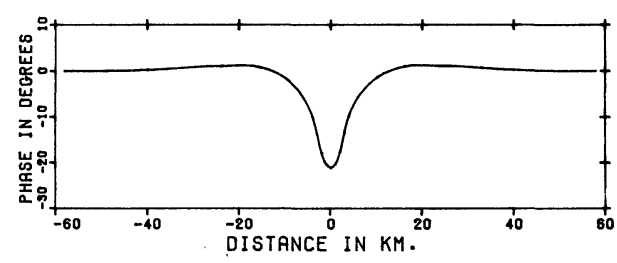

NORMALIZED PHASE OF EX/HY

Fig. 3. As Fig. 2 but for profile 2. 


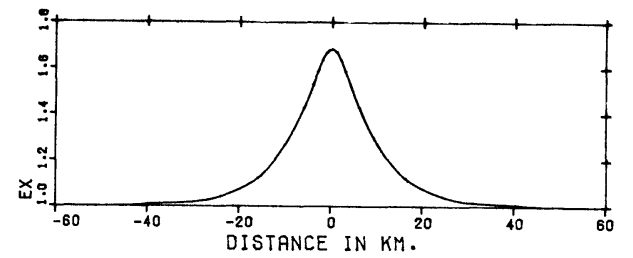

NORMALIZED AMPLITUDE EX

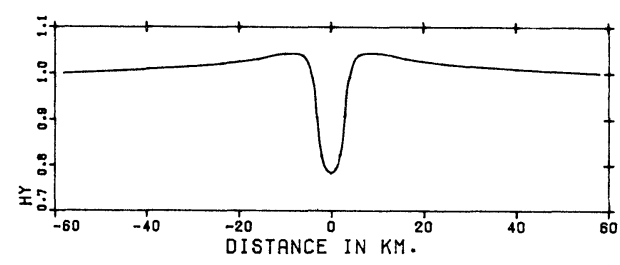

NORMALIZED AMPLITUDE HY

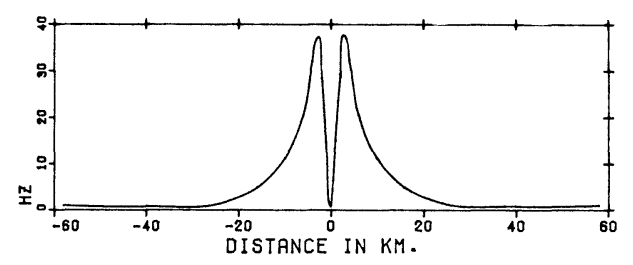

NORMALIZED AMPLITUDE $\mathrm{HZ}$

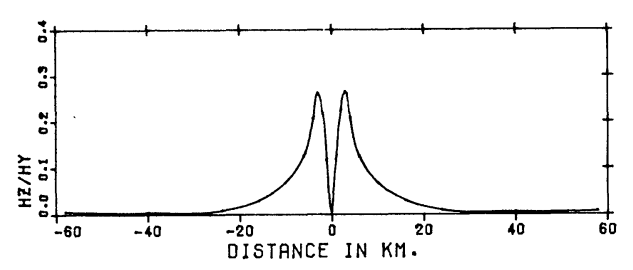

AMPLITUDE $\mathrm{HZ} / \mathrm{HY}$

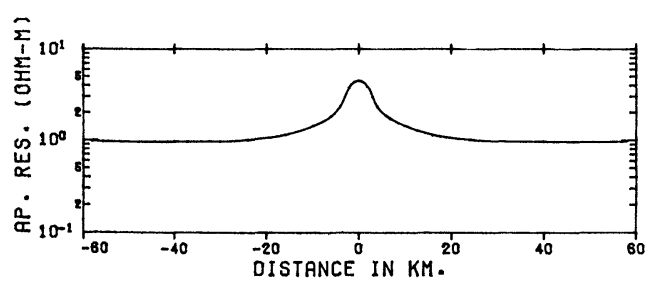

APPRRENT RESISTIVITY

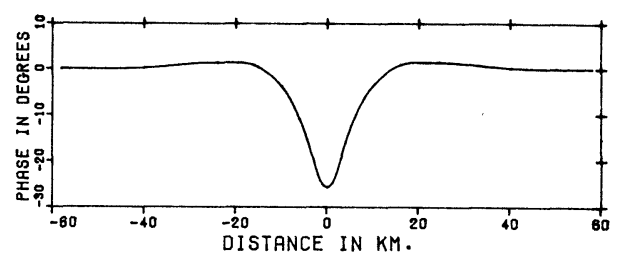

NORMALIZED PHASE OF EX

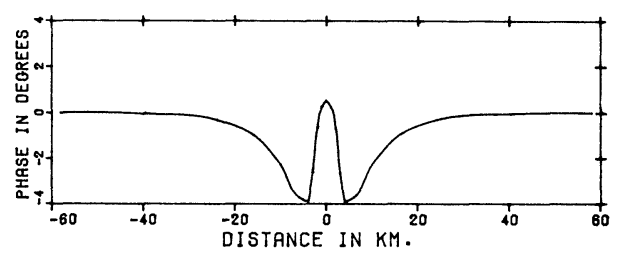

NORMALIZED PHASE OF HY

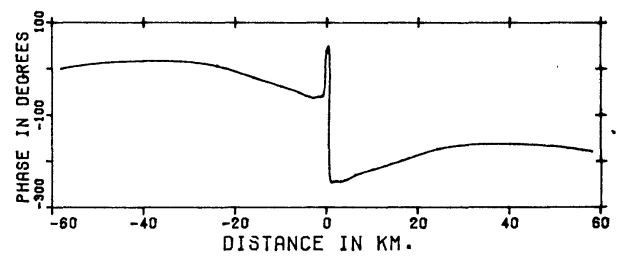

NORMALIZED PHASE OF $\mathrm{HZ}$

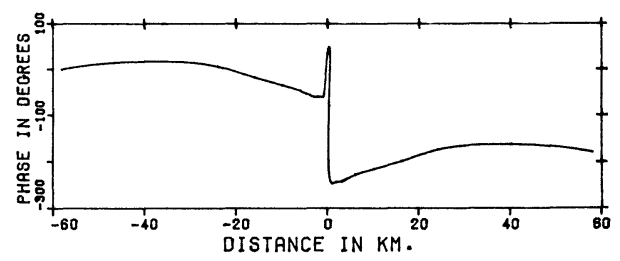

NORMALIZED PHASE OF HZ/HY

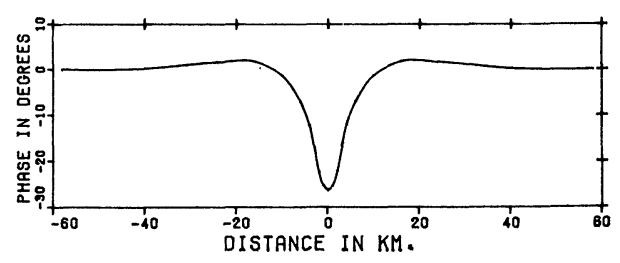

NORMALIZED PHASE OF EX/HY

Fig. 4. As Fig. 2 but for profile 3. 
nearly constant the apparent resistivity $\left(\rho_{a}\right)$ curve is nearly constant at a value of $1 \mathrm{ohm}-\mathrm{m}$. The value differs from the actual resistivity of $0.25 \mathrm{ohm}-\mathrm{m}$ because of the long period used since the calculated apparent resistivity value is influenced by the lower conductivity region. The phases are nearly constant, varying by only a few degrees, for all profiles except $H_{z}$ and $H_{z} / H_{y}$. As would be expected, the phase of $H_{z}$ and therefore $H_{z} / H_{y}$ shifts by $180^{\circ}$ over the center of the anomaly, which is consistent with the change of sign of $H_{z}$ there.

The second set of profiles, taken along the line $x=-2 \mathrm{~km}$ is given in Fig. 3 and clearly illustrates the effects of the anomaly. $\left|E_{x}\right|$ increases over the anomaly and $\left|H_{y}\right|$ decreases. $\left|H_{z}\right|$ and $H_{z} / H_{y}$ markedly increase over the anomaly. The apparent resistivity now shows a small increase in value over the anomaly and its value is two orders of magnitude below the actual resistivity value. The size of the anomaly is insufficient to cause an appreciable change in the apparent resistivity. The phase of the electric field, $E_{x}$, changes by a maximum of 23 degrees over the anomaly when compared to the phase of the electric field at $-58 \mathrm{~km}$. The phase of the apparent resistivity is similar to the electric field
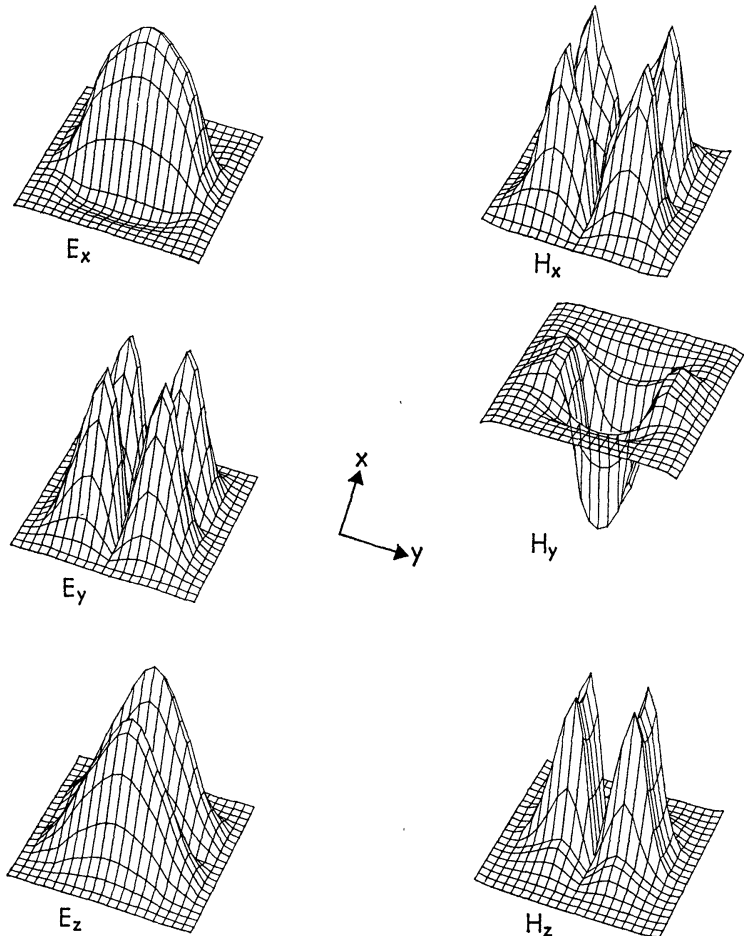

$\mathrm{H}_{y}$

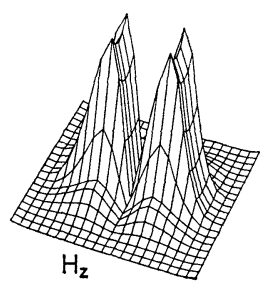

Fig. 5. Three-dimensional profiles of $\left|E_{x}\right|,\left|E_{y}\right|,\left|E_{z}\right|,\left|H_{x}\right|,\left|H_{y}\right|$ and $\left|H_{z}\right|$ for one-dimensional source field. 
phase since the phase of $H_{y}$ is nearly constant. Again the phases of $H_{z}$ and $H_{z} / H_{y}$ change by 180 degrees as the center of the anomaly is crossed. The peaks noted in these phases at the origin are artificial since the value of the vertical magnetic field is very small at this point.

The third set of profiles, taken across the center of the anomaly is given in Fig. 4. This set is almost identical with the second set of profiles except that $\left|E_{x}\right|$ is more sharply peaked as would be expected.

Three-dimensional amplitude profiles of the six electromagnetic field components are presented in Fig. 5. The decrease in the $\left|E_{x}\right|$ component off the anomaly in the $-X$ direction due to the concentration of charge at the boundary of the anomaly is clearly evident. Over the anomaly $\left|E_{x}\right|$ increases while $\left|H_{y}\right|$ decreases. $\left|H_{z}\right|$ increases over the edges of the anomaly in the $+Y$ and $-Y$ directions. Near the corners of the anomaly both $\left|E_{y}\right|$ and $\left|H_{x}\right|$ increase as would be expected due to bending of the fields in these regions. The vertical electric field, $\left|E_{z}\right|$, increases on both sides of the anomaly in the $+X$ and $-X$ directions. This is because the current is deflected vertically by the island structure.

\section{Two-Dimensional Source Field}

A two-dimensional source field can be obtained from a non-uniform sheet current flowing above a layered conductor. The general boundary conditions for this case were outlined by PrICE (1950). Again in the present work, only the $E$-polarization case is considered since it is most easily adapted to the formulation of the solution for the electromagnetic fields of a non-uniform sheet current source. For this case both the horizontal magnetic field component, $H_{y}$, and the vertical magnetic field component, $H_{z}$, will be present in the solution for a layered conducting subsurface.

In this work, a sheet current of rectangular intensity distribution which is $1,440 \mathrm{~km}$ in width and centered $840 \mathrm{~km}$ from the center of the L-shaped embedded anomaly (Fig. 1b) is considered. This sheet current flows at a height of $110 \mathrm{~km}$ above the surface of the conductor. The conductivity anomaly is embedded in a two-layered semi-infinite conducting region with a plane boundary which is chosen to represent an average continental vertical conductivity distribution. The first layer is $50 \mathrm{~km}$ in thickness and of conductivity $0.01 \mathrm{mho} / \mathrm{m}$. The second layer extends to infinity and has a conductivity of $0.1 \mathrm{mho} / \mathrm{m}$. The embedded anomaly is L-shaped, as in Fig. $1 \mathrm{~b}$, and has a depth of $3 \mathrm{~km}$. The conductivity of the anomaly is $0.0001 \mathrm{mho} / \mathrm{m}$ and the calculation is done for a frequency of $0.1 \mathrm{~Hz}$.

The boundary conditions for the finite difference mesh were determined 
Electromagnetic Induction in Three Dimensional Structures for Various Source Fields

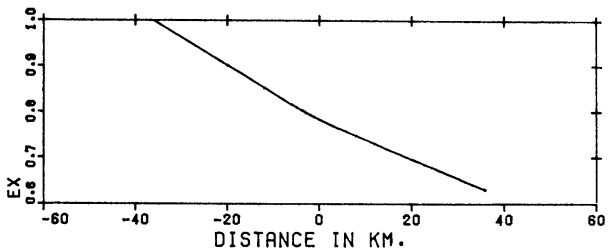

NORMALIZED AMPLITUDE EX

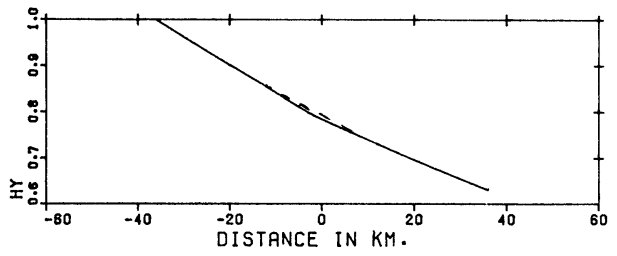

NORMALIZED AMPLITUDE HY

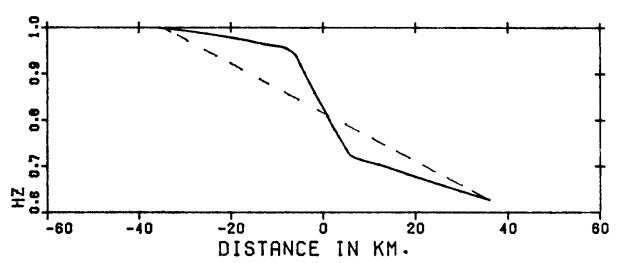

NORMALIZED RMPLITUDE $\mathrm{HZ}$

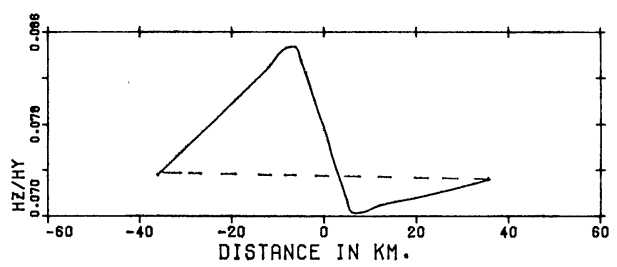

AMPLITUDE $\mathrm{HZ} / \mathrm{HY}$

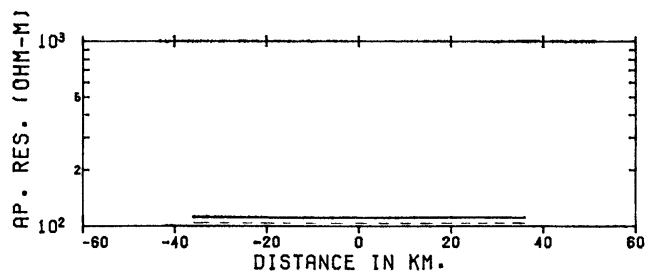

APPARENT RESISTIVITY

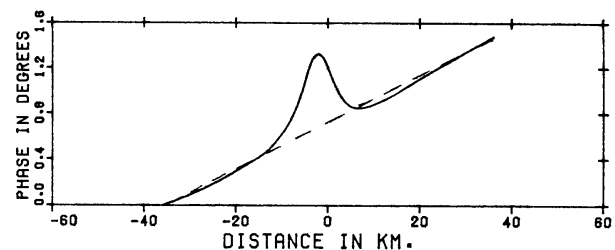

NORMALIZED PHASE OF EX

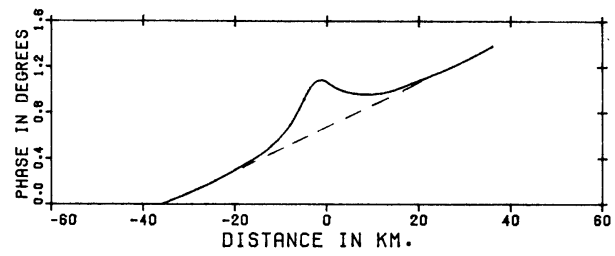

NORMALIZED PHASE OF HY

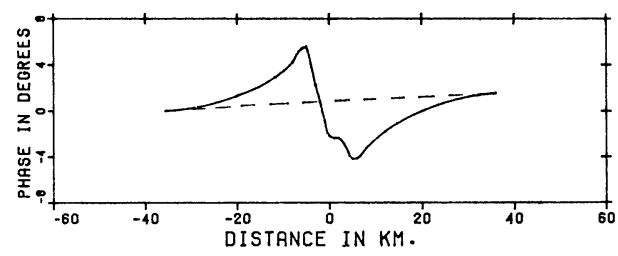

NORMALIZED PHASE OF $\mathrm{HZ}$

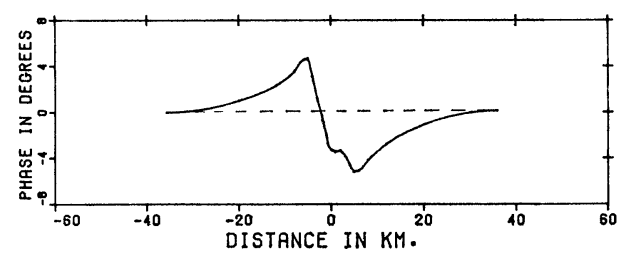

NORMALIZED PHASE OF HZ/HY

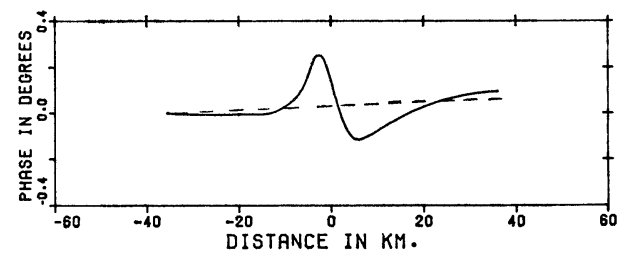

NORMALIZED PHASE OF EX/HY

Fig. 6. $\left|E_{x}\right|,\left|H_{y}\right|,\left|H_{z}\right|, H_{z} / H_{y}$ and $\rho_{a}$ profiles with normalized phases for two-dimensional source field and profile 1. $\left|E_{x}\right|,\left|H_{y}\right|$ and $\left|H_{z}\right|$ are normalized at $-38 \mathrm{~km}$. - 


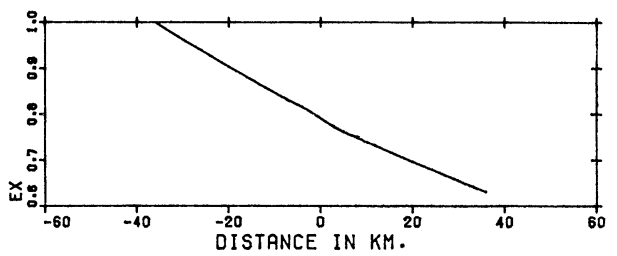

NORMALIZED AMPLITUDE EX

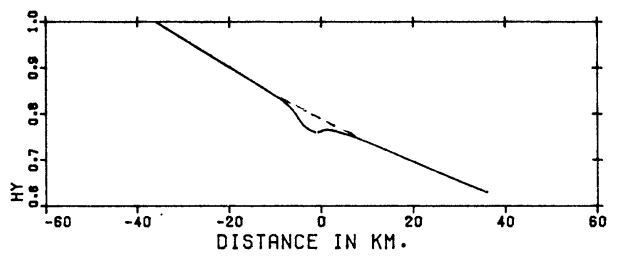

NORMALIZED AMPLITUDE HY

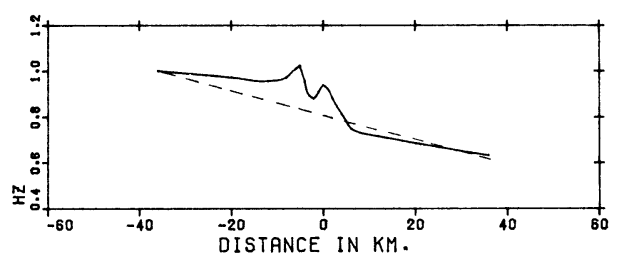

NORMALIZED AMPLITUDE $\mathrm{HZ}$

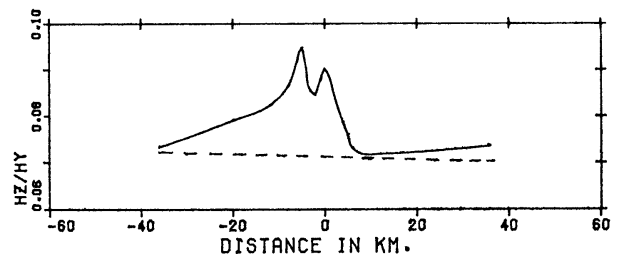

AMPLITUDE $\mathrm{HZ} / \mathrm{HY}$

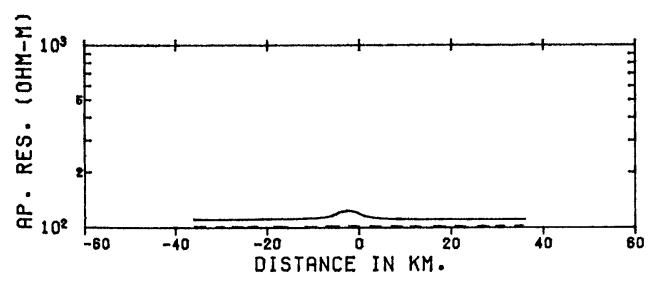

APPARENT RESISTIVITY

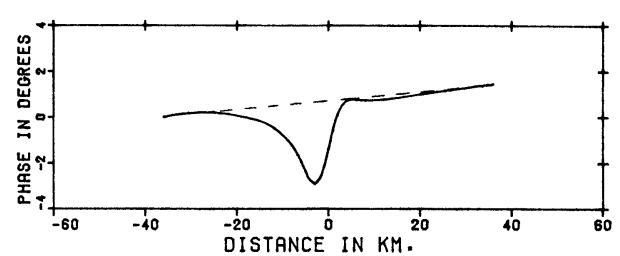

NORMALIZED PHASE OF EX

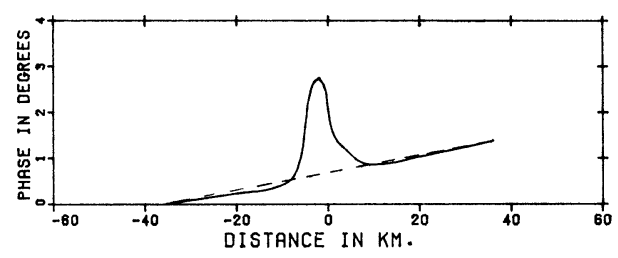

NORMALIZED PHASE OF HY

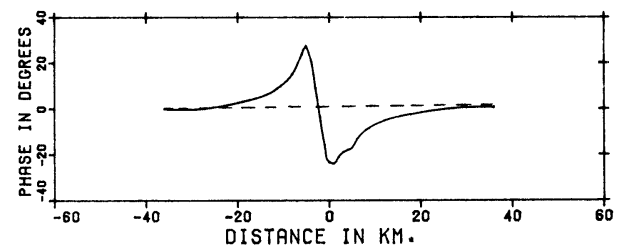

NORMALIZEO PHASE OF $\mathrm{HZ}$

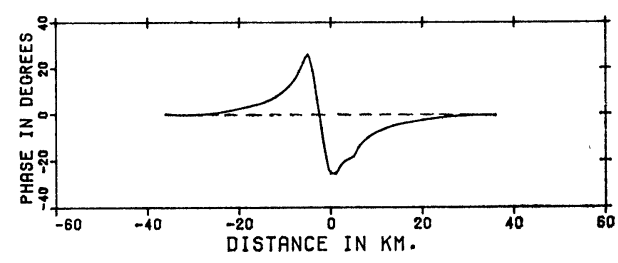

NORMALIZED PHASE OF HZ/HY

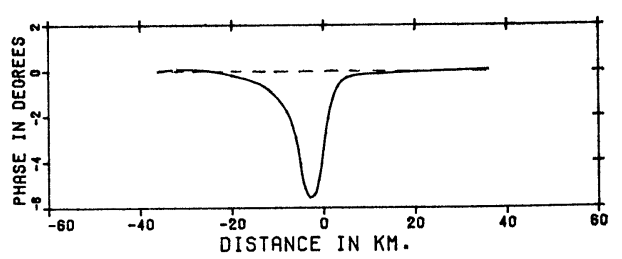

NORMAL IZED PHASE OF EX/HY

Fig. 7. As Fig. 6 but for profile 2 . 


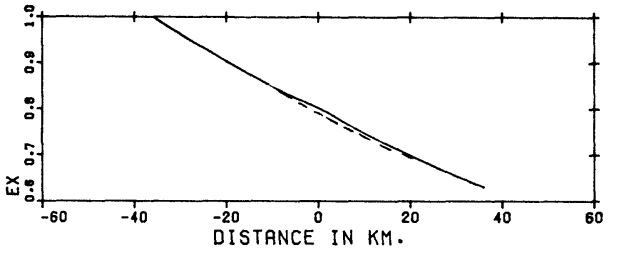

NORMALIZED AMPLITUDE EX

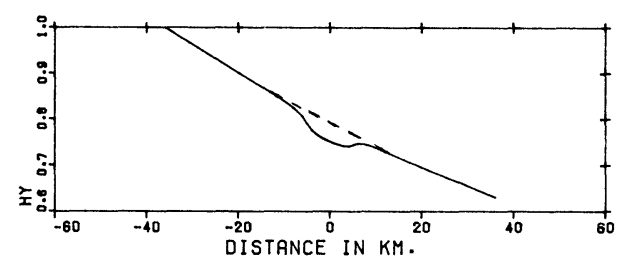

NORMALIZED AMPLITUDE HY

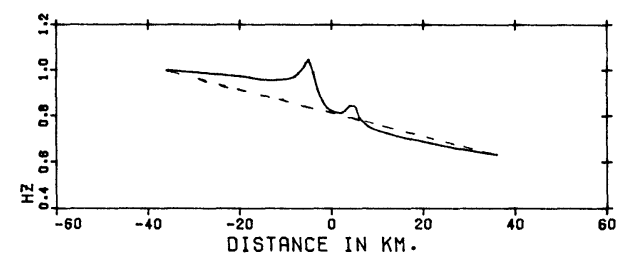

NORMALIZED AMPLITUDE $\mathrm{HZ}$

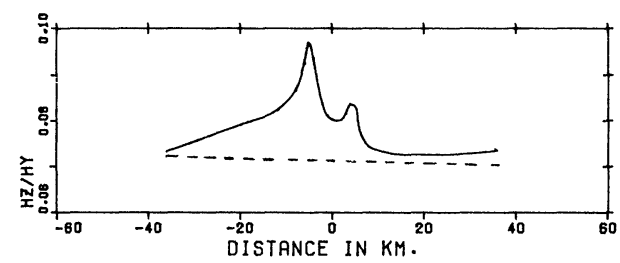

AMPLITUDE HZ/HY

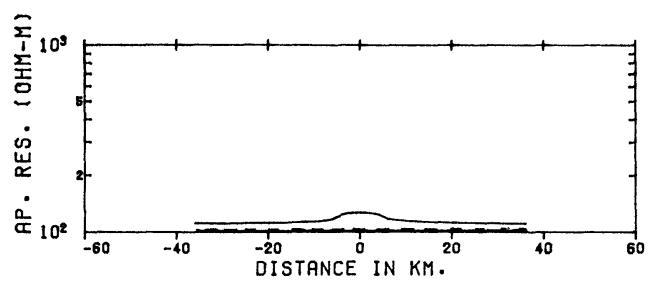

APPARENT RESISTIVITY

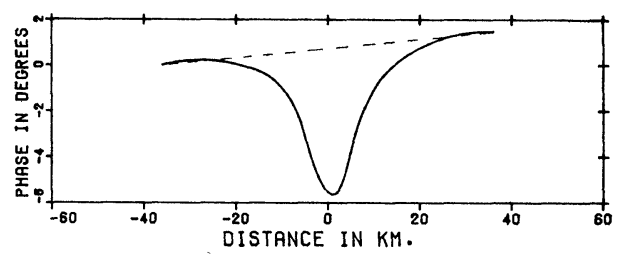

NORMALIZED PHASE OF EX

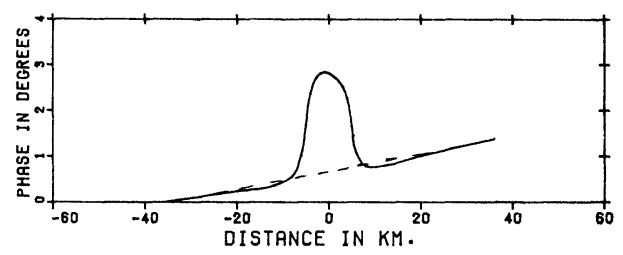

NORMALIZED PHASE OF HY

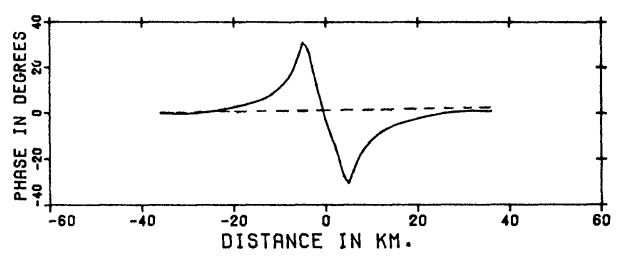

NORMALIZED PHASE OF $\mathrm{HZ}$

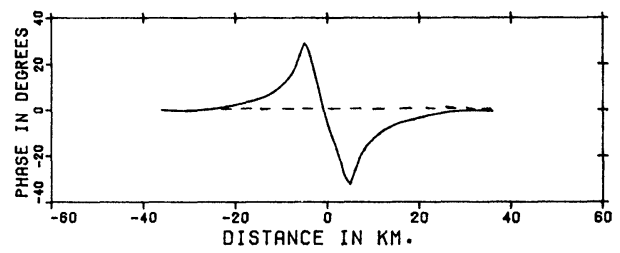

NORMALIZED PHASE OF HZ/HY

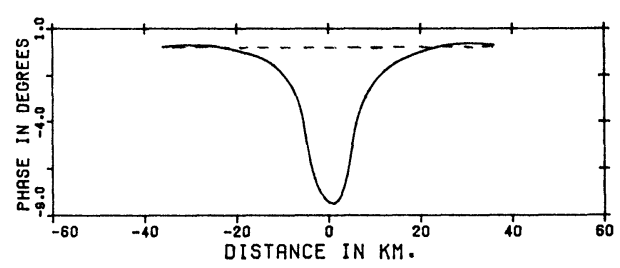

NORMALIZED PHASE OF EX/HY

Fig. 8. As Fig. 6 but for profile 3 . 
by using the general method for two-dimensional sheet current distributions over layered conductors of HibBs and Jones (1976 a, b). These values were then substituted into the numerical technique of Lines and Jones (1973) and the electromagnetic field solutions obtained.

The three profile positions chosen for this anomaly are shown in Fig. 1b. The profiles are calculated over the range $-38 \mathrm{~km}$ to $+38 \mathrm{~km}$ in the $Y$ direction. Each field component or ratio with the exception of the apparent resistivity and $H_{z} / H_{y}$ is normalized with respect to its value at $-38 \mathrm{~km}$. All phases are normalized to zero at $-38 \mathrm{~km}$.

The first set of profiles which is taken $6 \mathrm{~km}$ from the center of the anomaly in the $-X$ direction is shown in Fig. 6. The sloping character of the normal field (dashed curve) due to the non-uniform source is clearly evident in the $\left|E_{x}\right|$, $\left|H_{y}\right|$ and $\left|H_{z}\right|$ profiles. Since the conductivity contrast is only 100 to 1 , the effect of the anomaly at this profile position is not evident for $\left|E_{x}\right|$ and $\left|H_{y}\right|$ at this frequency. Also, as will be seen later, the rapid change in the source field tends to screen the effect of the anomaly when the fields are normalized. The effect of the anomaly can be clearly seen in the $\left|H_{z}\right|$ and $H_{z} / H_{y}$ profiles. The apparent resistivity is constant for this profile and corresponds in value to the conductivity of the upper layer. The phases are sensibly uniform for all quantities for this profile position although the expanded scale of the diagram amplifies any change in phase.

The second set of profiles (Fig. 7) is taken $2 \mathrm{~km}$ from the center of the anomaly in the $-X$ direction. These show effects due to the anomaly. The anomaly is just evident in the $\left|E_{x}\right|$ profile while in the $\left|H_{y}\right|$ profile the dipping of the profile due to the anomaly is apparent. The $H_{z} / H_{y}$ profiles clearly show the effect of the anomalous structure. As before, the apparent resistivity profile shows a slight increase due to the anomaly. However, the size of the structure is too small for the apparent resistivity to have a value equal to the resistivity of the anomaly which is $10,000 \mathrm{ohm}-\mathrm{m}$. A noticeable change in the phases of both $H_{z}$ and $H_{z} / H_{y}$ is now evident over the anomaly while the other phases remain relatively constant.

The third set of profiles, given in Fig. 8, is much the same in form as the second set of profiles. However, the effect of the anomaly is apparent over a greater distance than before due to the increase in the width of the anomaly at this profile position. Also, more clearly evident in the $\left|H_{z}\right|$ and $H_{z} / H_{y}$ profiles is the induction effect of the $H_{z}$ component of the source field. The peaks in the $\left|H_{z}\right|$ profile are not symmetric and differ in character.

In Fig. 9 the three-dimensional amplitude profiles of the six electromagnetic field components are presented. The figures are distorted near the edges since a non-uniform grid was used in calculating the field solutions while a uniform 

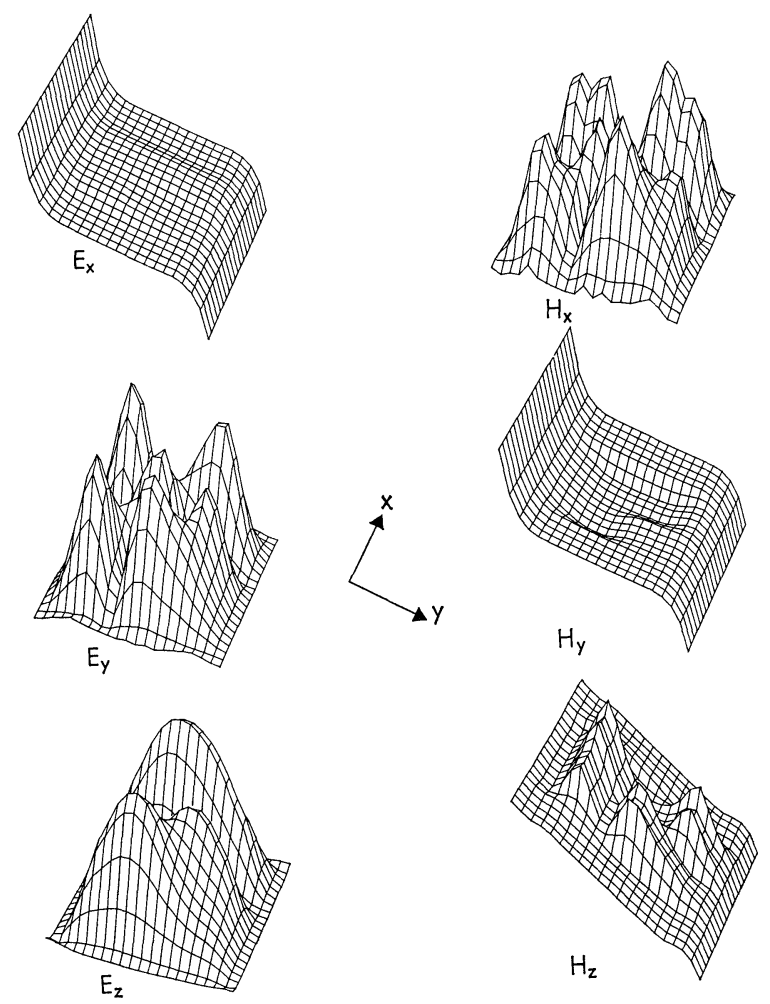

Fig. 9. As Fig. 5 but for the two-dimensional source field.

grid was used in the three-dimensional presentation. The anomalous electric field in the $X$ direction, $\left|E_{x}\right|$, is barely discernible due to the rapid spatial change of the source field. All the other electromagnetic field components clearly show the effects of the non-symmetric structure. The corners of the structure are identified with large increases in $\left|E_{y}\right|$ and $\left|H_{x}\right| \cdot\left|H_{y}\right|$ dips over the structure and the wide edges of the structure are outlined by $\left|H_{z}\right|$ and $\left|E_{z}\right|$.

\section{Three-Dimensional Source Field}

A three-dimensional source field can be obtained by using a horizontal magnetic dipole as a source. The boundary conditions for such a source were formulated by WAIT (1962).

In the model considered a horizontal magnetic dipole was placed $17 \mathrm{~km}$ in the negative $Y$ direction from the center of the anomaly, see Fig. 1c, at a height of $150 \mathrm{~km}$ above the surface of the anomaly. The dipole is polarized in the negative $Y$ direction. The conductivity anomaly is embedded $0.5 \mathrm{~km}$ beneath the 


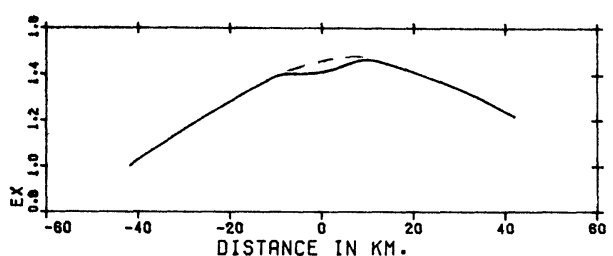

NORMAL IZED AMPLITUDE EX

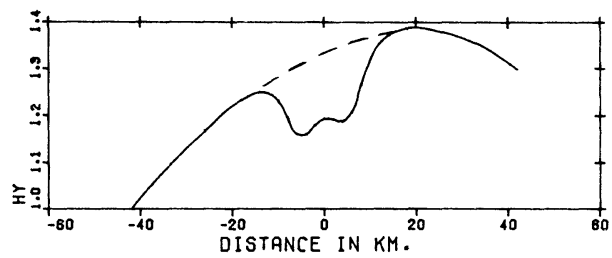

NORMAL IZED AMPLITUDE HY

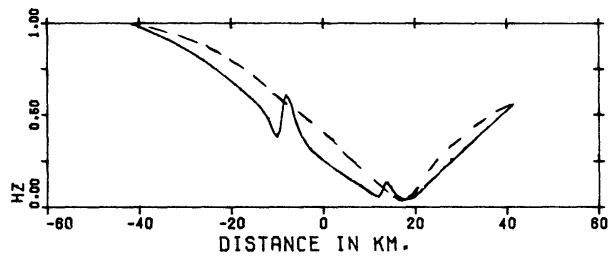

NORMALIZED AMPLITUDE $\mathrm{HZ}$

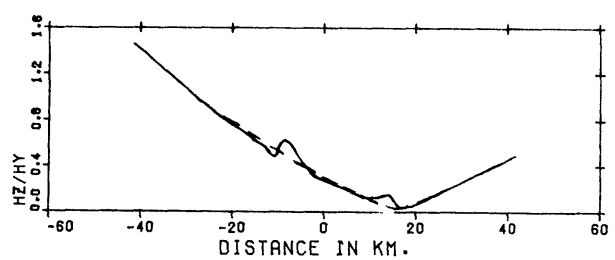

AMPLITUDE HZ/HY

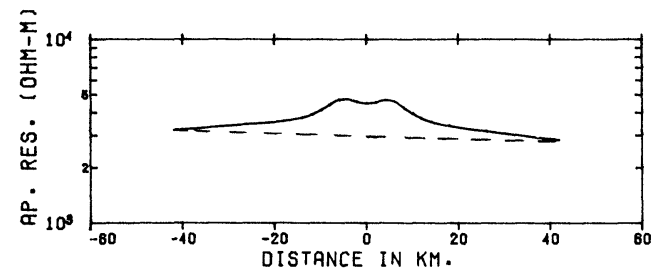

APPARENT RESISTIVITY

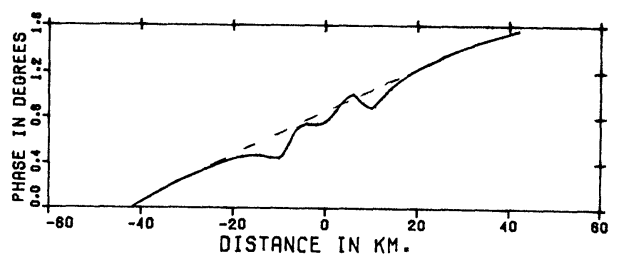

NORMALIZED PHASE OF EX

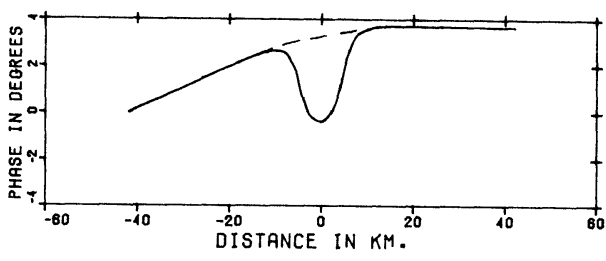

NORMAL IZED PHASE OF HY

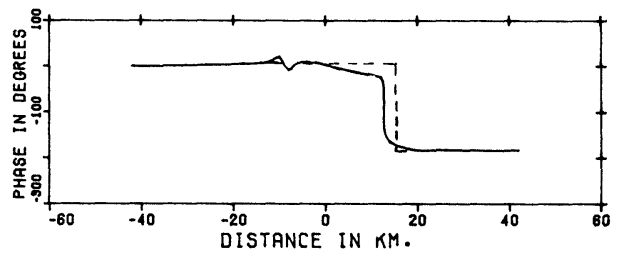

NORMAL IZED PHASE OF $\mathrm{HZ}$

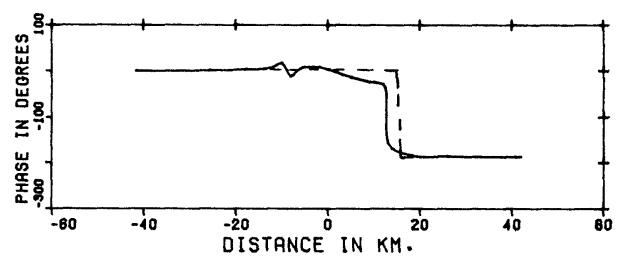

NORMAL IZED PHASE OF $\mathrm{HZ} / \mathrm{HY}$

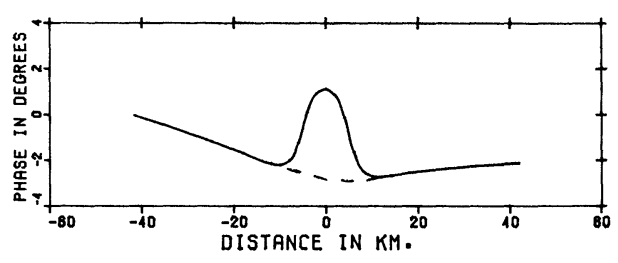

NORMALIZED PHASE OF EX/HY

Fig. 10. $\left|E_{x}\right|,\left|H_{y}\right|,\left|H_{z}\right|, H_{z} / H_{y}$ and $\rho_{a}$ profiles with normalized phases for three-dimensional source field and profile $1 .\left|E_{x}\right|,\left|H_{y}\right|$ and $\left|H_{z}\right|$ are normalized at $-42 \mathrm{~km}$. $\ldots$, total field; -..--, normal field. 


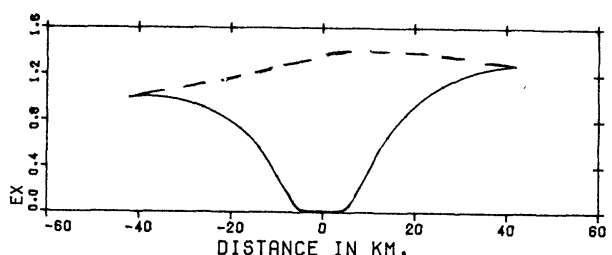

NORMALIZED AMPLITUDE EX

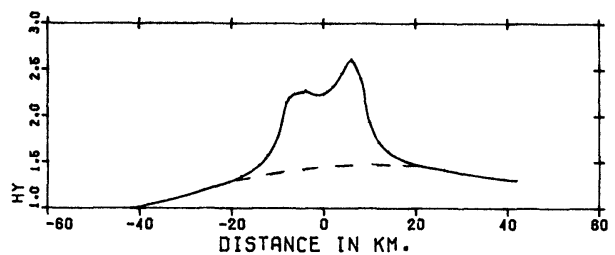

NORMAL IZED AMPLITUDE HY

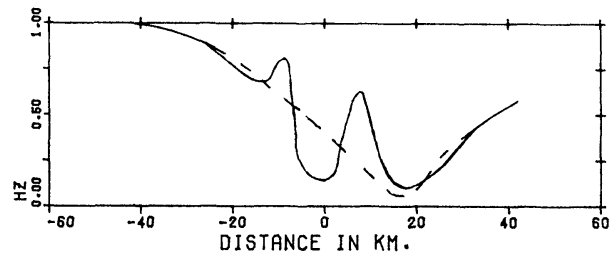

NORMALIZED AMPLITUDE $\mathrm{HZ}$

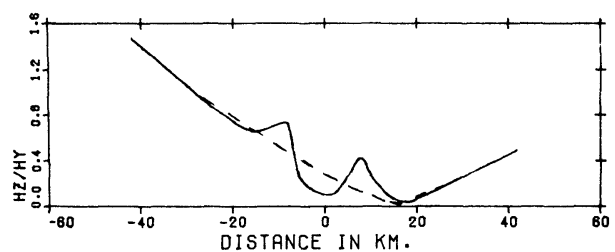

AMPLITUDE $\mathrm{HZ} / \mathrm{HY}$

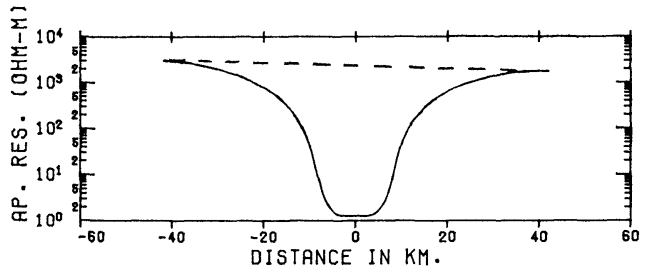

APPARENT-RESISTIVITY

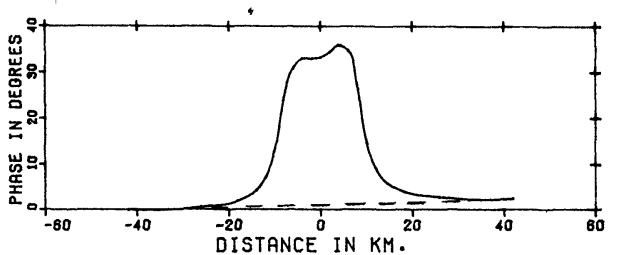

NORMALIZED PHASE OF EX

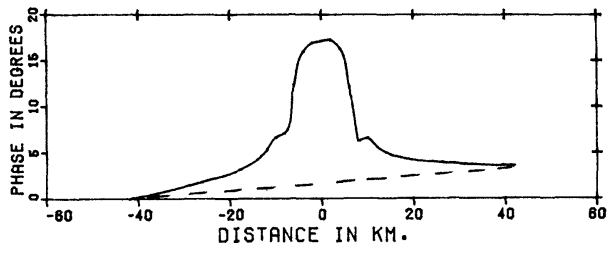

NORMALIZED PHASE OF HY

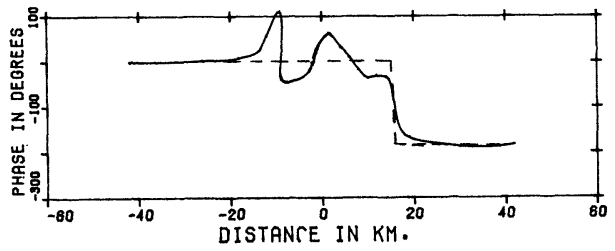

NORMALIZED PHASE OF $\mathrm{HZ}$

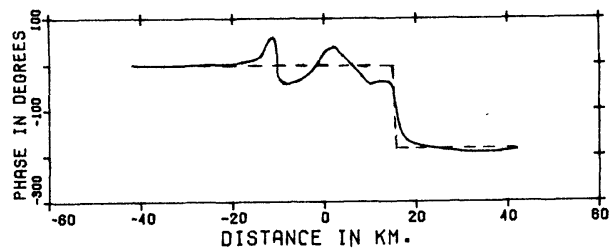

NORMALIZED PHASE OF HZ/HY

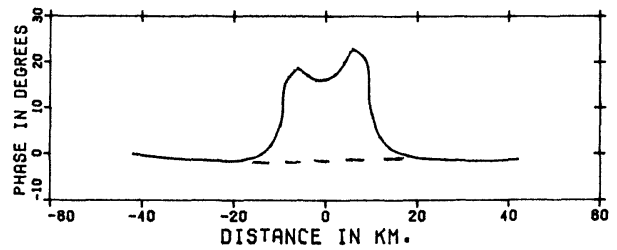

NORMALIZED PHASE OF EX/HY

Fig. 11. As Fig. 10 but for profile 2. 
surface in a two layered semi-infinite conducting region with a plane boundary. The first layer is $128 \mathrm{~km}$ in depth and of conductivity $0.21 \times 10^{-3} \mathrm{mho} / \mathrm{m}$. The second layer is of infinite depth and has conductivity $0.8 \mathrm{mho} / \mathrm{m}$. The embedded anomaly (see Fig. 1c) is $16 \mathrm{~km}$ square and $4 \mathrm{~km}$ thick and has the same conductivity as the lower layer. A transition zone $4 \mathrm{~km}$ thick of conductivity $0.21 \times$ $10^{-1} \mathrm{mho} / \mathrm{m}$ surrounds the anomaly on all sides except the top.

The boundary conditions were calculated by using the method of RAMASWAMY (1973) for the horizontal magnetic dipole over a layered conductor. These values were then substituted into the finite difference mesh of LINEs and Jones (1973) and the electromagnetic field solution obtained. This calculation was carried out for a frequency of $0.075 \mathrm{~Hz}$.

The two profile positions for this anomaly are shown in Fig. 1c. The profiles are calculated over the range $Y=-42 \mathrm{~km}$ to $Y=+42 \mathrm{~km}$. Each field component or ratio with exception of the apparent resistivity and $H_{z} / H_{y}$ is normalized with respect to its value at $-42 \mathrm{~km}$. All phases are normalized to zero at $-42 \mathrm{~km}$.

The first set of profiles which is taken $24 \mathrm{~km}$ from the center of the anomaly in the $-X$ direction is shown in Fig. 10. The non-uniformity of the source
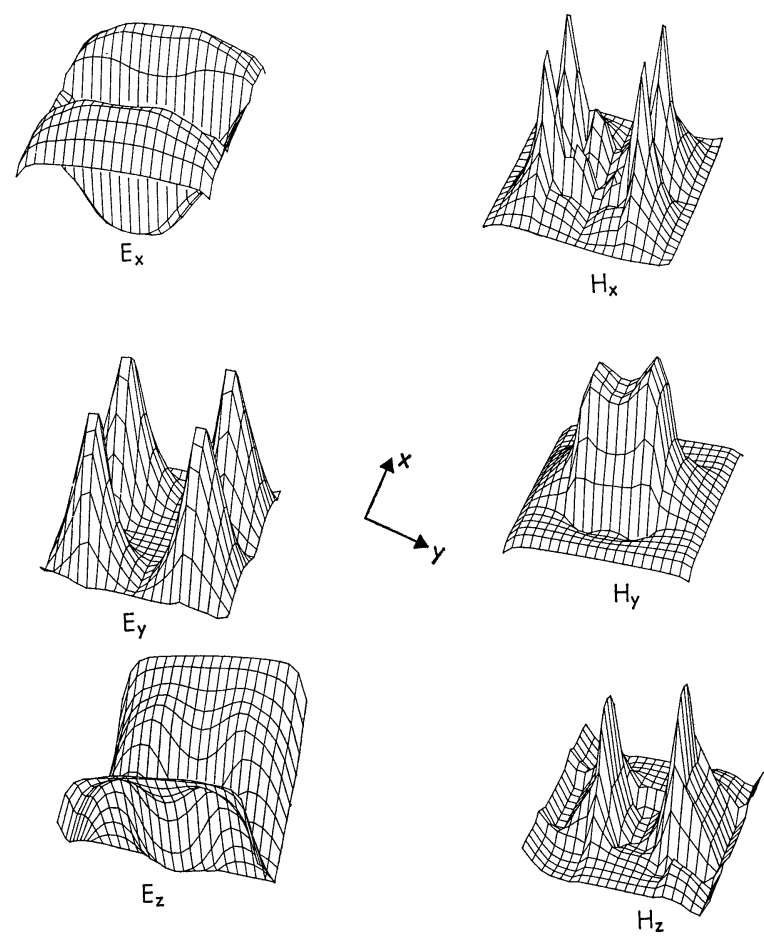

Fig. 12. As Fig. 5 but for the three-dimensional source field. 
field is clearly evident in all the amplitude profiles. For this frequency and conductivity contrast the anomalous structure is evident at this distance away from the anomaly. There is an increase due to charge concentration on the anomaly boundary in the $\left|E_{x}\right|$ profile as well as a depression in the $\left|H_{y}\right|$ profile. The boundaries of the anomly are shown in the $\left|H_{z}\right|$ and $H_{z} / H_{y}$ profile by slight peaks. The apparent resistivity profile exhibits a slight increase, opposite to what would be expected, because of the increased electric field in that region caused by the charge concentration. The phases are nearly constant with the exception of the $H_{z}$ and $H_{z} / H_{y}$ phases which go through a change of 180 degrees as the vertical component of the source field goes through zero.

The second set profiles, taken across the center of the anomaly, is shown in Fig. 11. In these profiles the effect of the anomaly is clearly displayed in all ampitude components and their phases. A marked depression in the $\left|E_{x}\right|$ profile is evident. $\left|H_{y}\right|$ increases rapidly over the anomaly and the $\left|H_{z}\right|$ and $H_{z} / H_{y}$ profiles exhibit two sharp spikes over the edges of the anomaly. The apparent resistivity varies from approximately the value of the first layer resistivity to the value of the resistivity of the anomaly. Increases in phase are noted for $E_{x}, H_{y}$ and $\rho_{a}$ while the phases of $H_{z}$ and $H_{z} / H_{y}$ change by $180^{\circ}$ over the anomaly and also as the source field goes through zero.

In Fig. 12 the three-dimensional amplitude profiles of the six electromagnetic field components are presented for the three-dimensional source field case. A large depression in $\left|E_{x}\right|$ is present over the anomaly while just outside the anomaly the field increases slightly due to the varying surface charge on the boundary between the conductivity regions. $\left|H_{y}\right|$ increases over the anomaly and the corners of the anomaly are marked by increases in $\left|H_{x}\right|$ and $\left|E_{y}\right|$. The sides of the anomaly are defined by $\left|E_{z}\right|$ and $\left|H_{z}\right|$. The peaks of $\left|H_{z}\right|$ are not of equal height because of the non-uniform source field.

\section{Conclusions}

The profiles of the field components and ratios along with their respective phases exhibit the character of the source fields away from the the anomaly. Over the anomaly the profiles are affected by both the conductivity configurations and the source fields. Excess charge on the boundary of the anomaly is indicated in the electric field, $\left|E_{x}\right|$, profiles.

This work was supported by the National Research Council of Canada. The authors wish to thank Dr. V. Ramaswamy for the computer program used to calculate the fields due to a horizontal dipole and for his assistance in using the program. 


\section{REFERENCES}

Cagnaiard, L., Basic theory of the magnetotelluric method of geophysical prospecting, Geophysics, 18, 605-635, 1953.

HibBs, R.D. and F.W. Jones, The calculation of the electromagnetic fields of a sheet current with arbitrary spatial intensity distribution over a layered half-space, 1. The general Method and Results, Geophys. J.R. Astron. Soc., 46, 433-452, 1976a.

HibBs, R.D. and F.W. Jones, The calculation of the electromagnetic fields of a sheet current source with arbitrary spatial intensity distribution over a layered half-space, 11 . The computer program and its application, Geophys. J.R. Astron. Soc., 46, 453-465, 1976 b.

JONES, F.W. and L.J. PASCOE, The perturbation of alternating geomagnetic fields by threedimensional conductivity inhomogeneity, Geophys. J.R. Astron. Soc., 27, 479-485, 1972.

Jones, F.W. and A.T. Price, The perturbations of alternating geomagnetic fields by conductivity anomalies Geophys, J.R. Astron. Soc., 20, 317-334, 1970.

LiNes, L.R. and F.W. Jones, The perturbation of altenating geomagnetic fields by three-dimensional island structures, Geophys. J.R. Astron. Soc., 32, 133-154, 1973.

PRICE, A.T., Electromagnetic induction in a semi-infinite conductor with a plane boundary, $Q$. J. Mech. Appl. Math., 3, 385-410, 1950.

RAMASWAMY,V., Electromagnetic fields of a horizontal dipole situated above and within a two layered earth, Ph.D. thesis, Univ. of Victoria, 1973.

TreumanN, R., Three dimensional analysis of the elecromagnetic induction problem of magnetic variation and magnetotelluric sounding, Geomagn. Aeron., 10, 844-853, 1970.

WaIt, J.R., Electromagetic Waves in Stratified Media, Pergamon Press, 1962.

WeIDELT, P., Electromagnetic induction in three-dimensional structures, J. Geophys., 41, 85109, 1975. 